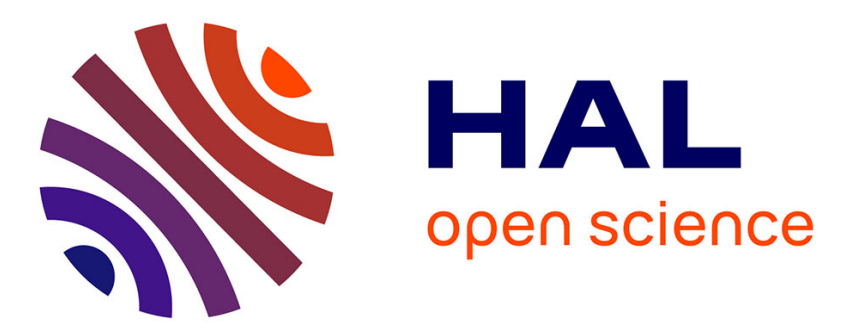

\title{
Fleurs de rhétorique, Buissons Ardents et Arbres de Jessé: autour de quelques comparaisons, métaphores et paraboles dans Perceforest
}

Christine Ferlampin-Acher

\section{- To cite this version:}

Christine Ferlampin-Acher. Fleurs de rhétorique, Buissons Ardents et Arbres de Jessé: autour de quelques comparaisons, métaphores et paraboles dans Perceforest . Le Moyen Français, 2007, 59, p. 205-231. hal-01845748

\author{
HAL Id: hal-01845748 \\ https://hal.science/hal-01845748
}

Submitted on 20 Jul 2018

HAL is a multi-disciplinary open access archive for the deposit and dissemination of scientific research documents, whether they are published or not. The documents may come from teaching and research institutions in France or abroad, or from public or private research centers.
L'archive ouverte pluridisciplinaire HAL, est destinée au dépôt et à la diffusion de documents scientifiques de niveau recherche, publiés ou non, émanant des établissements d'enseignement et de recherche français ou étrangers, des laboratoires publics ou privés. 
Fleurs de rhétorique, Buissons Ardents et Arbres de Jessé:

autour de quelques comparaisons, métaphores et paraboles dans Perceforest

\section{Christine Ferlampin-Acher \\ Université de Rennes}

L'importance de l'analogie dans les modes de pensée médiévaux semble promettre une forte présence de la métaphore et de la comparaison dans le vaste ensemble romanesque qu'est Perceforest, ce qui, joint au nombre des études modernes portant sur la métaphore ${ }^{1}$ et la primauté que lui accordent Jakobson et Ricoeur' ${ }^{2}$, confirmerait à l'occasion que l'homme est bien comme le voulait Nietzsche, « un animal métaphorique $»^{3}$ Or bien que, dans les manuscrits qui, au XVe siècle, nous conservent le roman de Perceforest $^{4}$, le narrateur, dans l'épisode du siège de Malebranche (livre I, f. 225), évoque la

1 La richesse de la bibliographie consacrée à la métaphore est telle que nous renonçons à établir une note sur ce sujet. La remarque d'Umberto Eco ne peut que décourager: "Un autre paradoxe -et non des moindres- auxquels on s'expose en abordant cette réflexion, c'est que l'on s'aperçoit très vite que, sur les milliers de pages écrites à propose de la métaphore, la plupart n'ajoutent rien de nouveau aux deux ou trois premiers concepts fondamentaux énoncés par Aristote. En effet, on a dit très peu de choses d'un phénomène sur lequel il semble que tout ait été dit. L'histoire du débat sur la métaphore, c'est l'histoire d'une série de variations autour de quelques tautologies, voire d'une seule: "La métaphore est cet artifice qui permet de parler métaphoriquement" (Sémiotique et Philosophie du langage, trad. Paris, 1988, p. 140). Ma seule excuse sera donc Perceforest et sa prolixité féconde.

2 P. Ricoeur, La métaphore vive, Paris, Seuil, 1975; R. Jakobson, "Two Aspects of Language and two Types of Aphasia Disturbances", Fundamentals of Language, La Haye, Mouton, 1956, trad. franç., "Deux aspects du langage et deux types d'aphasie", in Essais de linguistique générale, chap. II, Paris, Ed. de Minuit, 1963.

3 Cité par J. J. Murphy, Rhetoric in the Middle Ages, University of California Press, 1977, p. 11.

4 Ce travail s'appuie sur les éditions suivantes: Perceforest (le roman de), début de la première partie, éd. J. H. M. Taylor, Genève, T.L.F., 1979 ; deuxième partie, éd. G. Roussineau, Genève, T. L. F., vol. I, 1999 et vol. II, 2001; troisième partie, éd. G. Roussineau, Genève, T.L.F., vol. I, 1988 ; vol. II, 1991 ; vol. III, 1993 ; quatrième partie, éd. G. Roussineau, Genève, T.L.F., 1987. Pour les parties inédites, les références que j'ai utilisées renvoient, pour la fin du livre I, au manuscrit BnF fr. 345, pour le livre V au BnF fr. 348, et pour le livre VI au manuscrit Arsenal 3493 et 3494. 
nécessité de florir les fais ${ }^{5}$, métaphores et comparaisons sont relativement rares dans ce volumineux récit: des pages et des chapitres entiers en sont dépourvus. Il n'est pas possible de mener ici une étude exhaustive du sujet: nous l'interdisent la longueur de l'oeuvre et l'absence (provisoire) d'édition complète (malgré les travaux de J. Taylor et de G. Roussineau). Limitent aussi nos objectifs les problèmes liés aux définitions divergentes et à la diversité des travaux modernes sur le sujet, ainsi que la confrontation, souvent décevante, des arts poétiques médiévaux, héritiers de la rhétorique antique, et des pratiques littéraires contemporaines. Simplifiant le problème, je définirai la comparaison et la métaphore à partir de la relation d'analogie qu'elles instaurent entre un comparant et un comparé (qui n'est pas nécessairement exprimé dans le cas de la métaphore), le comparant désignant un référent virtuel (sans quoi il n'y a pas figure, comme dans « il parle comme son père »), l'opposition entre comparaison et métaphore reposant sur le fait que la comparaison est instrumentalisée par un outil (comme, tel) ${ }^{6}$. Après une approche descriptive et après avoir constaté et tenté d'expliquer la rareté relative de ces fleurs de rhétorique que sont les comparaisons et les métaphores dans Perceforest, je m'intéresserai à la critique de l'image métaphorique comme deception (entre autre à travers la référence au Buisson Ardent qui sert à peindre la Beste Glatissant) puis, à travers une série de trois songes présentant des arbres, je verrai comment le roman glisse vers la parabole: l'image devient une source de connaissances, fiable et

Pour un résumé complet du roman, voir J. Lods, Le roman de Perceforest, Genève Droz, Lille Giard, 1951, p. 24-ss et les articles de L. F. Flutre, "Etudes sur Le Roman de Perceforest", Romania, t. 70, 1948-9, p. 474-ss ; Romania, t. 71, 1950, p. 374-ss et 482-ss ; Romania, t. 74, 1953, p. 44-ss ; Romania, t. 88, 1967, p. 475-ss ; Romania, t. 89, 1968, p. 355-ss ; Romania, t. 90, 1969, p. 341-ss ; Romania, t. 91, 1971, p. 189-ss. Ces articles analysent le roman en suivant le plus souvent l'édition de 1528, proche des manuscrits. On consultera avec profit l'article de J. Taylor, "The Fourteenth Century: Context, Text and Intertext", dans The Legacy of Chrétien de Troyes, t. I, éd. N. J. Lacy, D. Kelly, K. Busby, Amsterdam, 1987, p. 267-332. 5 Mais pour ce que je ne vous puis pas tout recorder par paroles pour ce que je vous feroie ennuy an ce et que la matiere n'a mestiers a prolonger combien je florisse les fais et prolonge par paroles ung pou plus long que ne les treuvent ou latin pour estre ung pou plus plaisans a oyr sans riens adjouster aux fais de nouvel, car se je disoie cellui cellui tua, cellui et cellui navra l'autre et cellui ost desconfi tel autre et m'en passasse aussi briefvement que fait le latin, on vous auroit tantost tout compté, et si n'y auriez ja en plaisance a l'oyr ne deduit. Et se je floris les fais par parolles plaisans selon les fais qui sont touchiez en gros au latin, ce n'est pas merveille, car tout aussy tost porroit il anoyer par trop brief passer que par trop demourer.

6 C. Fromilhague, Les figures de style, Paris, Nathan, 1995, p. 74-75. Le sens du terme metaphore au Moyen Age est d'ailleurs assez large et confond souvent métaphores et comparaisons: voir A. Planche, "Naissance et sens du mot metaphore dans le Roman de la Rose", dans Miscellanea Mediaevalia, Mélanges Philippe Ménard, Paris, Champion, 1998, p. 1029-1042. 
cautionnée par la translatio imperii (d'Alexandre à Arthur), la translatio fidei (des païens au Christ), et la translatio studii (d'Ovide au roman). La translatio, au sens rhétorique du terme ${ }^{7}$, n'intéresserait donc pas l'auteur comme ornement topique, mais comme outil permettant de penser et de dire l'Histoire, tant dynastique que providentielle.

\section{Présence mesurée des comparaisons et métaphores dans Perceforest}

Si l'on rencontre dans Perceforest des comparaisons bien identifiables, comme dans le cas d'un géant qui se met à bruire comme tonnoirre (1. IV, p. 119), et des métaphores, comme lorsque le sabbat des sorcières est appelé laide feste et si lait tournoy (II, I, p. 219), il n'empêche que l'usage qui est fait de ces figures est modéré. Elles signalent surtout l'excellence, au sujet de la beauté des femmes et de l'héroïsme de la fleur de chevalerie. Mais ces figures ne sont ni nombreuses (relativement à l'ampleur de Perceforest) ni développées. Le relevé des comparaisons et des métaphores s'enrichit pourtant si l'on prend en compte deux types d'expressions plus fréquentes dans le roman, qui correspondent à un élargissement définitionnel: les expressions avec degré de l'adjectif et les identifications atténuées et subjectives.

Le degré des adjectifs se substitue souvent aux « similitudes » au sens strict du terme: Blanche est plus blanche que neige (1. II, t. I, p. 191), la barbe de Dardanon est plus blanche que fleurs de liz (II, I, p. 232), Sarra a la chair plus blanc que fin alebastre mis a point de vermeil (1. I, chap. XXVII). Dans ces cas d'adynaton ${ }^{8}$, le degré de l'adjectif vise en fait à établir non pas un étalonnage mais une analogie entre le comparant et le comparé qui tous deux sont blancs à l'extrême. Le comparant est une référence indépassable, rien n'est plus blanc que la neige, le lis ou l'albâtre. L'expression est hyperbolique et renvoie à une impossibilia. Ces degrés combinent donc l'hyperbole et la comparaison et sont interchangeables avec des comparaisons au sens strict du terme: dans le livre III au sujet de deux pierres, on trouve à la fois deux expressions avec un degré (l'une estoit une esmeraude plus verde que l'erbe qui

\footnotetext{
${ }^{7}$ Sur ce terme, voir par exemple J. J. Murphy, op. cit., p. 11 et passim.

${ }^{8}$ L'adynaton est classé parmi les figures de pensée, et les comparaisons et les métaphores parmi les tropes : il existe néanmoins un continuum de l'un aux autres, dont témoignent les permutations fréquentes, dans Perceforest par exemple.
} 
croit emmy le pré, et l'autre estoit ung ruby plus ardant et alumé que feu III, I, p. 245), et dans le songe de Neronès qui double cette évocation, se lisent deux comparaisons simples (vermeille comme sang et verde comme herbe III, II, p. 342).

Pourquoi ces formulations sont-elles si nombreuses ? Pourquoi dire d'une femme qu'elle est plus belle qu'une fée et non qu'elle est belle comme une fée ? Ces expressions interviennent très souvent dans des épisodes merveilleux, où d'une part la surenchère est de règle ${ }^{9}$, et où d'autre part la merveille renvoie à l'indicible et passe volontiers par des impossibilia. On se rapproche alors de la métaphore dans la mesure où celle-ci, contrairement à la comparaison, repose sur un « conflit conceptuel $»^{10}$.

D’autres formulations, du type «Le poète est semblable au prince des nuées », passent par des opérateurs d'illusion, «sembler », " avoir l'air de », « on eût dit», dans un continuum qui va de l'identification atténuée à la vision subjective ${ }^{11}$ et incitent aussi à élargir les champs de la métaphore et de la comparaison. Les images sont alors souvent développées dans des propositions au subjonctif imparfait, qui entretiennent l'incertitude entre l'irréel du passé et le potentiel. Le cerf de Péléon suit les chevaliers ainsi que ce fust ung mouton (II, I, p. 34): certes, il n'est pas un mouton, mais, créature merveilleuse, il peut être soupçonné d'être un de ces hybrides étranges, qui comme la Beste Glatissant, hantent les forêts féeriques. La tempête fantastique qui se déclenche au château de Bruyant se print a croistre tellement qu'il sambloit que tous les ennemis d'enfer fussent ou chastel Bruiant et par les marescaige d'environ (...); il sambloit que tout fondist en ung tas (IV, p. 291). Ces expressions, envahissantes dans Perceforest, sont souvent inventives et ont certainement permis à l'auteur de se dégager de la topique comparative du type «blanche comme noif negiee ». Relevons, parmi bien d'autres exemples, au sujet du tournoi qui a lieu lors de la fête du Dieu Souverain: ce sambloit une bataille en l'air des tronchons qui se rencontroient (IV, 27). Dans ces emplois, sembler et estre avis sont en concurrence, le second insistant sur le regard incertain si fréquent dans les épisodes merveilleux.

9 Voir C. Ferlampin-Acher, Merveilles et topique merveilleuse, Paris, Champion, 2003, p. 75-ss.

10 Voir par ex. M. Prandi, Grammaire philosophique des tropes, Paris, Editions de Minuit, 1992, p. 18-ss. Si je reprends l'image relevée plus haut au sujet du rubis, "le rubis est du sang" repose sur une prédication contradictoire (le rubis, solide, ne peut être liquide comme le sang), qui disparaît si j’introduis "comme", qui impose une sélection des critères analogiques: "le rubis est comme le sang".

11 C. Fromilhague, op. cit., p. 77-78. 
Semblable (plusieurs poissons semblables a moutons cornus III, II, p. 273), a maniere de (la pointe que portent les poissons chevaliers est en maniere d'espee III, II, p. 274), de la façon de se rencontrent aussi.

La discrétion des métaphores et des comparaisons au sens strict, et parmi celles-ci des topoi, est donc compensée en partie par ces deux types de formulations. Elle doit aussi être relativisée dans la mesure où certains passages sont très riches en images des deux types. En effet, comparaisons et métaphores ont tendance à se concentrer dans les discours d'un registre élevé et dans les épisodes merveilleux.

Il n'est pas surprenant que les comparaisons et les métaphores soient très présentes dans les discours soutenus, qu'il s'agisse des harangues royales, des sermons prononcés par la Reine Fée ou par des preudommes (et en particulier Dardanon, II, I, p. 236-ss), des allocutions trompeuses et pompeuses d'Aroés l'enchanteur ${ }^{12}$, ou de l'éloge grandiloquent que le narrateur prononce au début du livre II (p. 221). Dans ces discours apparaissent des comparaisons et métaphores au sens strict, qui reprennent parfois des topoi bien connus. Dans les 25 lignes du $\$ 267$ du livre II, les nobles sujets de Perceforest regrettent son état dépressif, et associent le roi et la lumière, le roi et le miroir, la fumée et Fortune, l'impacience et les chaînes, les loups et les mauvais sujets (II, I, p. 152-153). Plus que la cohérence d'un unique réseau d'images (qui pourrait déboucher sur l'allégorique), ce qui est recherché, c'est une formulation kaléidoscopique, où interviennent plusieurs systèmes qui souvent se juxtaposent sans véritablement se combiner. Plus que la qualité du locuteur, c'est le sujet du discours qui appelle le niveau stylistique: un simple marinier parle très noblement et avec force images de la mort du roi Alexandre (II, I, p. 131) ${ }^{13}$. Le texte épistolaire est une variante de discours qui présente lui aussi des

\footnotetext{
${ }^{12}$ Sur ce discours, voir C. Ferlampin-Acher, Fées, bestes et luitons, Paris, Presses de l’Université Paris Sorbonne, 2002, p. 184-ss.

13 Citons aussi le « chastoiement » de la reine qui exhorte Bétidès à ne pas épouser une Romaine (il ne fait pas bon estraindre estrange boiel au sien IV, p. 70), le sermon où Perceforest rejette le polythéisme (la vraye lumiere qui est le Dieu Souverain IV, p. 76), le discours que Gadifer fait au début du livre II où il développe l'image du roi pasteur et évoque les maladie du peuple (II, I, p. 2), le planctus émis par Gadifer blessé, où la métaphore de la lumière sert à évoquer le bon gouvernement (II, II, p. 52), l'intervention du narrateur qui regrette le faste des églises (III, III , p. 27).
} 
images nombreuses. La lettre posthume d'Alexandre ${ }^{14}$ est riche en métaphores et comparaisons: le roi et ses sujets sont vus comme le corps et ses membres, le monarque est une colonne, il se compare au paon qui se delite en faisant sa roe, mais comme regarde ses piez, tout son plaisir lui chiet (IV, p. 51).

A côté de ces discours marqués par une marqueterie d'images, d'autres, plus rares, filent la comparaison. Dans le discours où Perceforest dans le livre IV se plaint de ce que la chevalerie déserte sa cour, l'image du rossignol est longuement élaborée: « Je voy bien que la bonne chevalerie qui souloit ceans demourer puet estre de legier comparee au rouseignol. Car telle est sa nature que des le prin tamps qu'il commence a soy enamourer de sa femelle, il devient tant joyeulx et moult bien chantant qu'il le fait bon ouyr (...). Mais il vous couvient entendre que quant celle chasse avra prins fin et que le rousseignol se sent au dessus de sa pourqieutte, il ne fera que siffler n'en lui n'avra joye ne deduit. En pareille maniere il m'est advis qu'il soit des chevaliers du Franc Palais ». Le roi développe sur plusieurs pages l'idée que les chevaliers, qui une fois la dame conquise, délaissent les tournois, sont comme les rossignols qui chantent pour conquérir la femelle et ne font plus ensuite que siffler. En réponse, la reine lui opposera une autre image, celle de la chasse. Ce retour métalinguistique sur une comparaison éculée est l'occasion d'une exploration de l'image ${ }^{15}$ : l'écart entre la topique et le renouvellement proposé se mesure à l'ampleur du développement.

Par ailleurs, en dehors des discours constitués, deux réseaux, renvoyant à un savoir, valorisé et nécessitant donc une expression choisie, sont nourris de métaphores: la médecine (au sujet de la blessure de Gadifer II, I, p. 136-ss ${ }^{16}$ ), et la généalogie ( II, I, p. 12 de si gentille extraction, p. 138 qui yssy de son sang et de sa gendre...).

A côté de ces figures qui sont des métaphores et des comparaisons clairement reconnaissables, on relève, concentrés dans les épisodes merveilleux, de nombreuses images qui sont des identifications

14 Cette lettre reprend une longue tradition qui au XIIIe siècle se retrouve dans les nombreuses lettres au style orné du Roman d'Alexandre en prose (éd. H. Fukui, Y. Otaka, et C. Ferlampin-Acher, Osaka, 2003).

${ }^{15}$ Pour Thibaut de Champagne, le rossignol meurt de trop chanter d'amour.

16 Par exemple: toutesfoiz furent les nerfz si orguilleux et si plains de grant desdaing pour le contraire qu'ilz eurent au commencement que le roy ne peult estre gary (p. 138), l'indignacion et la felonnie que les nerfz avoient encontre leur garissement (p. 146). On notera la personnification, qui peut se justifier par une relation analogique de type synecdoque (entre la partie et le tout, l'organe et le corps dans son ensemble) et de type métonymie (entre le physique et le psychique). 
approximatives et plus ou moins subjectives. Elles sont en général plus inventives que les comparaisons topiques. Si « Malaquin chainglant comme quarrain d'arbalestre » (III, I, p. 85) est une comparaison (au sens strict) relativement banale, celle qui le fait entendre au milieu d'ung tres grant bruit (qui) venoit parmy la forest comme se ce fust un puissant cheval qui eust lyé en travers sus sa selle ung grant tinel et puis venist de toutes sa force courant et abatant tout ce qu'il rencontroit (III, I, p. 81) est nettement plus originale.

Strictes, marquées par un degré adjectival ou une identité approximative, les comparaisons et les métaphores sont des moyens privilégiés pour exprimer l'étrangeté et la diversité de la merveille qui, créature de l'écart, bouleverse les catégories et rejoint l'impossible. La pluralité comparative autour de la merveille sert souvent à rendre la diversité physique des monstres hybrides: la Beste Glatissant est décrite avec des groupes prépositionnels qui en font une composition improbable, même si chaque image, isolée, peut être référentielle (teste de serpent, corps de lyon, queue de lyon, mais aussi dens (...) en maniere d'un loup III, II, p. 215). De même les poissons de la fontaine Bouillant sont a testes serpentines, ils ont des piés a maniere d'asne, ils sont grans comme un saulmon, de couleur plus noire que arement, plus noirs que meures (1. IV, p. 166-167).

Ces images ont souvent une valeur axiologique, en fonction d'une symbolique préétablie et simple, qui oppose le noir et le blanc, le beau et le laid, l'obscurité et la lumière. C'est ainsi que nous rencontrons, parmi bien d'autres merveilles, un taureau bruyant comme fouldre (II, I, p. 194), un poulain plus noir que meure, un dragon volant aussi comme tempeste de mer (t. II, I, p. 340) et rapide comme ung effondre (p. 341), mais aussi des fées au teint de lis ou d'ivoire, à la blondeur lumineuse ${ }^{17}$. Dans ces expressions, les comparants sont souvent interchangeables. Au Pin de 1'Estrange Merveille, Gadifer est recouvert par les mauvais esprits d'une fumée diabolique: son haubert est plus noirs que arement, son cheval plus noirs que dyable, son écu plus noir que charbon (III, I, p. 21); les trois références pourraient permuter sans problème. Ces comparaisons reposent sur un système symbolique approximatif et incomplet: si la blancheur du teint des fées s'oppose à la noirceur des mauvais esprits, la tempeste bruyante associée à ceux-ci n'a pas d'équivalent féerique musical et harmonieux. Par ailleurs le critère esthétique peut être trompeur: la comparaison plus blancz que fin yvoire, qui en général renvoie à une

\footnotetext{
${ }^{17}$ Voir C. Ferlampin-Acher, Merveilles et topique merveilleuse, Paris, Champion, 2002, p. 141-ss.
} 
féerie bienveillante, est aussi employée, nous y reviendrons, au sujet des défenses d'un sanglier monstrueux (II, I, p. 47).

Les comparaisons par identification subjective sont aussi des outils privilégiés de l'écriture merveilleuse qui passe par une vision entravée ${ }^{18}$ : sambler, estre advis supposent un regard et une subjectivité. Dans certains cas, l'impression est prise en charge par un personnage dans un discours. Le Blanc Chevalier, s'adressant à un esprit maléfique, s'exclame: «Vous me samblez comme ung grant barreau de fer alumé et bouté en eaue et retiré hors ainchois qu'il soit estaint, tant grant friente et fumee sault il de vous » (III, I, p. 152). Le plus souvent néanmoins, l'absence de complément tend à associer le lecteur à cette identification: Et vous prommés que ce porcq menoit tel bruit en son venir que ce sambloit fourdre ( III, I, p. 294); le cerf de Péleon est aussy blanc que neige et sy semble de ses cornes que ce soit ung grant cheste ramus (II, I, p. 32) ${ }^{19}$. D'autre part, la concentration de comparaisons hétérogènes autour d'une merveille suggère des interprétations diverses de celle-ci, au service de la polysémie incertaine de la merveille ${ }^{20}$. Si les mauvais esprits qui attaquent Gadifer au Pin de 1'Estrange Merveille (III, I, p. 11-12) font une noise (...) comme fourdre, si ce sont de noirs esperis comme ethiopiens, s'ils sont noirs comme arrement, s'ils sont en samblance de chevaliers (p. 14), c'est qu'ils sont peut-être la foudre (interprétation météorologique), Ethiopiens (nous sommes renvoyés aux Images $d u$ Monde), de simples chevaliers, voire de l'encre, des mots (interprétation poétique). L’éclatement des comparaisons nourrit la polysémie merveilleuse ; le feuilleté de sens que génère la merveille -quand elle est merveilleuse- repose, plus sur des images ponctuelles, tout juste suggérées, que sur des figures filées.

Finalement la merveille ne tient que par la juxtaposition d'images, difficilement référentielles. C'est pourquoi il est particulièrement significatif que le terme figure, qui remonte à figura dont le sens rhétorique est bien attesté tant chez Quintilien que Pline ou Sénèque, et qui a aussi en ancien français une valeur rhétorique, serve très généralement à désigner les esprits diaboliques, revenants et démons, qui hantent les nuits de Perceforest. La merveille est d'abord une figure (rhétorique), une fiction

\footnotetext{
${ }^{18}$ Voir C. Ferlampin-Acher, Merveilles et topique merveilleuse, op. cit., p. 91-170.

${ }^{19}$ Avec estre advis, il est plus fréquent qu'un complément permette d'attribuer la vision (ce qui n'est que rarement le cas avec sembler): et tantost ö̈t autour de la salle une tourmente et une noise si grande de vent et d'autres plusieurs tourmentes qu'il estoit advis a Estonné que la sale deust cheoir (II, I, p. 216).

${ }^{20}$ Sur cette notion, Merveilles et topique merveilleuse, op. cit., p. 211-ss.
} 
poétique : les images sont donc loin d'être purement ornementales, elles sont les outils nécessaires d'une écriture merveilleuse dont l'orgueilleux objet est justement l'indicible. Paradoxalement, la merveille, inédite, sans pareil, incomparable, ne peut se dire qu'avec des comparaisons. La Grande Singesse amoureuse du Bossu de Suave est une merveilleuse singesse, grande et laide sans comparoison (IV, p. 65), la tempête que font les esprits maléfiques est grande sans compairoison qu'elle ne ressambloit a nulles autres (IV, I, p. 290). Il n'empêche que la Singesse parle un patois et « singe » les humains, tandis que la tempête menée par les mauvais esprits est régulièrement comparée à la foudre et à la fin du monde.

Plutôt que d'épuiser l'évidence des figures topiques (blanc comme neige, fier comme un lion), l'auteur a donc privilégié le tremblé d'une rhétorique de la merveille. C'est pourquoi nombre d'images méritent d'être analysées de près. Je ne retiendrai que deux exemples. Dans la première mention qui en est faite, les poissons chevaliers ont sur le dos comme un escu (III, II, p. 274), puis il est question, directement, du dur escu; de même ils ont d'abord une pointe, longue de une brasse et demie en maniere d'espee (III, II, p. 274), puis une espee ${ }^{21}$. Le passage de la comparaison à la métaphore, voire à l'identification, entretient l'incertitude autour de ces créatures, qui peuvent être soit des chevaliers, soit comme des chevaliers.

Autre exemple d'un traitement ambigu de l'image: l'ivoire qui sert de comparant pour décrire le sanglier qui blesse Gadifer. A première vue, on identifie une simple variante du topos «blanc comme ivoire ». Mais à y regarder plus en détail, il apparait que l'image repose sur une manipulation de la comparaison traditionnelle. En effet, la formule il avoit les.II. dens hors de sa gueule ung grant pié plus blancz que fin yvoire (II, I, p. 47), suivie par l'os (...) plus dur que dens d'yvoire ( p. 52) est paradoxale, car effectivement une dent de sanglier est en ivoire: l'image est attendue, mais surprend car au lieu d'une identification (une dent est bien en ivoire), nous avons une surenchère impossible (qui revient à dire cette dent est plus blanche et plus solide qu'une dent). D'autre part, il s'agit là d'une description: si l'ivoire est banal dans les portraits féminins au sujet des dents ou de la clarté du teint, ici, nous sommes face à un monstre, au sujet duquel l'ivoire surprend, d'une part parce qu'il est fin, ce qui renvoie, hors de propos, une idée de fragilité et de délicatesse, et d'autre part, parce qu'il est rattaché sur le mode de l'inadéquation (plus ... que) aux défenses, qui pourtant de fait sont en ivoire. Ces écarts laissent le

\footnotetext{
${ }^{21}$ Voir C. Ferlampin-Acher, Fées, bestes et luitons, op. cit., p. 300-ss.
} 
lecteur incertain: il faudra plusieurs centaines de folios pour comprendre, dans le dernier livre, que le monstre, comme pouvait le laisser pressentir l'ivoire, n'est pas purement négatif dans la mesure où la moelle de ses défenses permettra la guérison, quasi miraculeuse, du roi, en ces temps où s'annonce le Christ. Parallèlement, comme tout sanglier, cette bête a d'abord ces deux défenses proéminentes, en ivoire (II, I, p. 47), puis, blessée par le roi, elle conserve un tronçon d'épée fiché dans son front en maniere de corne (p. 52). Par la suite, le comparant et le comparé sont inversés, le monstre a ou front devant une corne de la moictié d'une espee (p. 110), qui en fait un double des poissons chevaliers ou de Bucéphale. Les cornes merveilleuses de ce monstre sont construites, progressivement, à force d'images, qui déplacent la topique ou qui glissent vers la métaphore puis la confusion référentielle.

Dans Perceforest, les comparaisons et métaphores topiques seraient donc délaissées au profit d'images plus souples, qui se fondent dans l'écriture; la vertu ornementale recule au profit d'une valeur poétique nettement plus profonde ; l'image est essentielle : la merveille est d'abord une figure.

La désaffection que connaissent les formulations topiques peut s'expliquer de plusieurs façons. Les arts poétiques les considèrent avec réserve. E. Faral note les réticences que la comparaison et la métaphore éveillent chez Matthieu de Vendôme, Evrard l'Allemand et Geoffroy de Vinsauf et il souligne que les romans antiques, par rapport à leurs modèles, suppriment de nombreuses images ${ }^{22}$. L'influence de ces écrits cléricaux (par ailleurs nettement antérieurs à Perceforest) sur les romans n'étant pas facile à cerner, cette explication est incertaine. Il est plus vraisemblable que Perceforest hérite de la défaveur que les portraits et les descriptions des romans en vers, riches en formulations topiques, connaissent dans les proses du XIIIe siècle ${ }^{23}$. Perceforest, vraisemblablement composé au

22 E. Faral, Les arts poétiques du XIIe et du XIIIe siècle, Paris, Champion, 1962, p. 68-ss.

23 Pourtant au XIIIe siècle, Brunet Latin, dans son Livre du Trésor valorise les couleurs de rhétorique, au service de l'amplificatio: la tierce coulour por acroistre tes dis est apelee comparison, et c'est la plus bele croissance et avenable que parleur facent. Mais ele est devisee en.II. manieres, car ou ele est coverte ou ele est descoverte. Et cele ki est descoverte se fait connoistre par.III. mos ki segnefient comparison, ce sont plus, mains, et autant. (...). La seconde maniere, ki est coverte, ne se fait pas connoistre a ses signes; ele ne vient pas en son abit, ains moustre une autre samblance dehors ki est si comme jointe a la verité dedens comme s'ele fust de la matire meismes. Raison comment: d'une home perreceux je dirai c'est une tortue, et d'un isnel je dirai c'est uns vens. Et sachiés que ceste maniere de parler est mout bele et mout bonne et cortoise et de 
$\mathrm{XIVe}$ siècle, repris au XVe siècle, aurait hérité sa réserve face aux comparaisons à la fois du roman et de la prose.

D'autre part, Perceforest se présente comme une chronique, comme en témoignent les prologues, les rubriques et les interventions du narrateur ${ }^{24}$. La vérité qu'il revendique impose à l'auteur d'écrire $a$ plain, droitement, c'est-à-dire en évitant ces écarts que sont les images. Comme Geoffroy de Monmouth, dont l'Historia Regum Britanniae est traduite relativement fidèlement au début du roman, l'auteur de Perceforest semble repousser la fable et ses mensonges ornés ${ }^{25}$ : certains épisodes suggèrent, comme nous le verrons dans la deuxième partie, une condamnation de l'image illusoire (condamnation qui passe par un va-et-vient, sans surprise car attesté par exemple dans les arts poétiques, entre l'image visuelle et l'image rhétorique).

Par ailleurs, la rareté des comparaisons et métaphores peut s'expliquer par le fait que la relation d'analogie, sur laquelle reposent ces figures, est déplacée dans Perceforest de l'elocutio à l'inventio et à la dispositio. Le projet de Perceforest d'écrire une préhistoire prémonitoire du monde du Graal conduit à la mise en place de jeux de miroirs entre les aventures racontées et celles d'un large intertexte arthurien. Ainsi les aventures de Gadifer, blessé, affolé de la cuisse, puis guéri, reprennent (sur le plan de l'invention) et annoncent (sur le plan de l'organisation du texte) celles du roi Méhaigné. Le transfert analogique participe ainsi à la mise en place d'une vision du monde fondée sur la translatio imperii et la translatio fidei et exprimée à travers un jeu complexe de réécritures analogiques. La nécessaire lisibilité du texte aurait imposé que les analogies purement ornementales soient rares. La translatio (au sens de comparaison $)^{26}$, si elle avait été combinée aux translationes imperii, studii et fidei aurait peut-être

bone sentence et mout le puet on trover es dis de sages home (Li Livres dou Tresor, éd. F. Carmody, Slatkine Reprints, Genève, 1975, III, 13). Le décalage entre pratique et théorie est aussi net qu'au XIIe siècle.

24 Voir C. Ferlampin-Acher, "Perceforest et le temps de l'(h)istoire", dans Dire et penser le temps dans l'historiographie médiévale (XII-XVe siècles), dir. L. Harf-Lancner et E. Baumgartner, Presses de la Sorbonne Nouvelle.

25 Geoffroy de Monmouth dans sa préface explique en effet: «Je n'ai pas fait moisson de propos fleuris dans les jardins d'autrui, je me suis contenté d'un style simple et personnel » trad. L. Mathey-Maille, Histoire des rois de Bretagne, Paris, Les Belles Lettres, La Roue à Livres, 1992, p. 26.

${ }^{26}$ Matthieu de Vendôme, reprenant une définition largement répandue, associe métaphore et translatio (metaphora est alicujus verbi usurpata translatio éd. E. Faral, op. cit., p. 172), tandis que très souvent l'ensemble des figures analogiques, et en particulier la métaphore, sont nommées translationes (comme chez Geoffroy de Vinsauf). 
brouillé la lecture. Nous verrons néanmoins dans la troisième partie que la translatio historique et religieuse redonne, dans certains passages, à l'image, et à la rhétorique, ses lettres de noblesse, tandis que l'on glisse de la comparaison ornementale et de la métaphore vers l'allégorie, dont la vogue est très forte à l'époque de Perceforest.

\section{Colores rhetorici, Buisson Ardent et illusion: l'image et ses deceptions}

Comme nous l'avons vu précédemment, l'image est fréquente dans des discours de registre élevé, à visée persuasive, qui peuvent être trompeurs, comme dans le cas d'Aroés, et dans les épisodes merveilleux, où, par exemple l'or des cheveux des demoiselles fées se confond dangereusement avec l'or des cheveux du Géant aux Crins Dorés (il sembloit que chascun cheveil fust un fil d'or II, II, p. 78). Les réserves que soulèvent l'image rhétorique peuvent se lire dans un certain nombre d'épisodes qui dénoncent les illusions des images visuelles, qui marquent le refus de la litote, et insistent sur le risque de prendre les métaphores au pied de la lettre. La Beste Glatissant et ses deceptions mortifères, Troÿlus découvrant le désir physique, entre euphémisme et hyperbole, et les métamorphoses qui prennent l'image au pied de la lettre nous serviront d'exemples.

La Beste Glatissant, ce formidable écran à fantasmes, illustre le danger des images illusoires ${ }^{27}$. Tout d'abord, comme bien des merveilles, composite, elle est décrite à travers une succession de similitudes qui marquent, de façon banale, son hybridation. Impossible à représenter ${ }^{28}$, elle n'est qu'un patchwork de comparaisons. Difforme, protéiforme et finalement informe, elle ne s'incarne monstrueusement que dans le verbe : c'est une figure. Par ailleurs, elle est certes inspirée par la panthère, la « leucrote » et le «scytalis » des bestiaires, mais l'apport original de Perceforest repose sur une métaphore synesthésique, moins repérable: cette créature a en effet des couleurs criardes. Elle est

\footnotetext{
${ }^{27}$ Voir C. Ferlampin-Acher, Fées, bestes et luitons, op. cit., p. 311-ss et "Les déceptions dans Perceforest: du fantosme au fantasme", dans Félonie, trahison, reniements au Moyen Age. Actes du troisième colloque international de Montpellier. Université Paul Valéry (24-26 novembre 1995), Les Cahiers du C.R.I.S.I.M.A., 3, 1997, p. 413-430.

${ }^{28}$ Voir sur ce point C. Ferlampin-Acher, «La peur du monstre dans le roman médiéval », dans Travaux de littérature, XVII, «Les grandes peurs », 2004, p. 119-134.
} 
multicolore, comme de nombreuses figures séduisantes et dangereuses, comme l'oiseau Willeri' ${ }^{29}$; l'un de ses modèles est la Bête qui porte en son sein des chiots glatissants. Bigarrée (les descriptions qui évoquent les jeux chromatiques de son dos sont somptueuses ${ }^{30}$ ), elle crie comme un braquet glatissant (III, II, p. 216). On passe du cri des chiots qu'elle porte, au glat qu'elle-même pousse, puis à celui de ses couleurs: la beste n'avoit couleur au col qui ne jectast son glat (III, II , p. 216). L'élément traditionnel, hérité, le monstre glatissant, est rattaché à la bigarrure, par la biais de la synesthésie, prise au pied de la lettre, des couleurs criardes.

Or chacun, comme Maronex dans le livre VI, peut voir sur son dos l'objet de ses désirs, tandis que les couleurs se mêlent les unes aux autres: adont encommencha celle beste a lever la teste et a drescer le col et estendre tant que le soleil se fery es couleurs qu'elle avoit alentour du haterel. Si ne pourriez croire comment la diversité des couleurs se print a entrecontrer par la forche du soleil et a enexer les unes couleurs dedens les altres et a reverberer les unes encontre les autres (...). Et tant estoit delitant aux regardans et merveilleux car quiconques s'i delitoit il y figuroit toutes les choses delicieuses ou son cour estoit le plus enclin (...). Bien lui fu advis qu'il voioit en celle reverberation de couleur une pucelle la plus belle a son jugement (...). Lors print mout fort a fantasier en celle plaisance et comme plus y fantaisoit, tant plus voioit il de merveilles dedens les couleurs et entre les differences aussi comme l'on fait en ung feu de dur charbon de nouvel alumé.

Cette expérience, sur laquelle l'auteur revient à plusieurs reprises, insiste sur le pouvoir qu'a le désir de créer des images, des fantasmes, et sur la séduction mortifère de ces apparitions qui permettent à la Beste de captiver des proies pour mieux les dévorer. Ces évocations peuvent être comprises comme la condamnation morale d'une fantasie échauffée et d'une rhétorique mal maîtrisée. Le vocabulaire (couleur, figurer) tout comme la description des images où les couleurs se mêlent, peut renvoyer aux images, aux figures, aux couleurs de rhétoriques, qui déplacent les frontières entre les notions et confondent les cheveux et l'or. Tout comme la métaphore opère par recatégorisation de la femme vers la fée, les couleurs de la Beste fusionnent: les frontières, tant chromatiques que conceptuelles, s'estompent.

29 Voir Cl. Roussel, « Le jeu des formes et des couleurs: observations sur la Beste Glatissant », dans Romania, t. 104, 1983, p. 49-82.

${ }^{30}$ Voir C. Ferlampin-Acher, Fées, bestes et luitons, op. cit., p. 311. 
Serait-ce d'ailleurs étonnant qu'un monstre caractérisé par sa voix soit le «porte-parole » de considérations poétiques?

Projections séduisantes, reflets trompeurs ${ }^{31}$ et figures rhétoriques fallacieuses vont donc de pair. Les images, tant rhétoriques que picturales, sont considérées avec méfiance. Dans le livre III, un long développement, qui sonne aussi comme une prémonition cistercienne, condamne les ymage et les pourtraiture dans les églises, afin qu'en aourant les personnes n'y applicquassent point leur ymaginacion, parquoy leur devocion fust moins valable (III, III, p. 26). Se mettre à créer des images, tant verbales que visuelles, est d'ailleurs souvent dans Perceforest un symptôme inquiétant, soit de folie, soit d'hypocrisie. Dans le livre V Norgal, plongé dans sa démence amoureuse, tout forsené, se met à faire des métaphores: il pense que desormés feroit le vent ses chevaleries sur lui et les rousses espines et chardons sergens des foretz leur arrestz et renderont gaige au departir comme des brebis trespassans (f. 204-ss). Les hypocrites Méléan et Nabon mettent en doute la loyauté de la femme de Margon pour la séduire (IV, p. 335-ss): leur discours au roi multiplie les métaphores (sans umbre de bien, venin, viande, dilicieux boires, cuer noircy), ils dénoncent (faussement) le mari comme ypocrite, dont le dedens et le dehors ne coïncident pas. C'est donc à mots couverts, avec des images, qu'ils l'accusent. Le roi cependant ne se laisse pas prendre à leur venin mauvais: avec sagesse, il retourne contre eux leur discours contre les hypocrites. Dans ce long épisode, le langage figuré est du côté de l'hypocrisie: Margon le loyal, lorsqu'il s'adresse au roi en toute bonne foi, n'use pas de rhétorique (p. 338). Lorsque plus loin, à tort, il sera convaincu de l'infidélité de sa femme, sous le coup de Jalousie et de Mélancolie, il se mettra à employer des images (p. 369-371), confusion des mots et confusion des cœurs allant de pair quand le désir physique, mal contrôlé (celui des deux felons), prend le pas sur la part morale et spirituelle en l'homme.

L’image, qu'il s'agisse de la Beste et de ses images visuelles, ou de Margon et de la rhétorique, naît d'un désir physique exacerbé : elle ne saurait être un voile pudique pour dire le sexe. Perceforest nie l'efficacité de l'euphémisme. Dans le livre III, Zélandine, La Belle au Bois Dormant, est tombée dans un sommeil léthargique, dont Troÿlus finira par la tirer amoureusement, non par un simple baiser,

\footnotetext{
${ }^{31}$ Voir C. Ferlampin-Acher, "Perceforest et ses miroirs aux alouettes", dans Miroirs et jeux de miroirs dans la littérature médiévale, textes réunis par F. Pomel, Rennes, Presses Universitaires de Rennes, 2003, p. 191-214.
} 
mais en concevant un enfant. Lorsque le chevalier interroge Vénus sur les moyens de guérir la demoiselle, la déesse répond en vers et en images:

Puis qu'eslissiez par la raiere

Le fruit ou gist la medecine

Garye seroit la meschine (III, III, p. 80).

La métaphore de la cueillette du fruit et le sens second et érotique de raiere (fente), attesté chez E. Deschamps $^{32}$, sont clairs, mais le jeune chevalier ne comprend pas. Vénus refuse d'expliciter ses paroles: Les vers n'ont point mestier de glose et elle poursuit son discours imagé: Amours trouvera la raiere/ Et Venus, qui scet la maniere/ Du fruit trouver, le queillera./ Nature le composera (p. 80).

Vénus est à la fois personnage (concret) et allégorie (abstraite): pour elle le passage de l'image à la chose est évident et immédiat. Mais pour Troÿlus, il n'en va pas de même ; il manie mal l'analogie entre le comparé et le comparant. D'une part il n'est pas capable de décrypter lui-même, et d'autre part la scène où enfin il passe à l'acte montre sa tendance juvénile à se satisfaire d'images. La description qui est faite de la demoiselle endormie rend compte de son regard ; il la contemple, comme dans un roman en vers ou dans une poésie, belle comme une deesse, tendre et vermeille comme une rose et de char blanche comme la fleur de lys (p. 87). Il ne voit pas la femme derrière l'image et reste passif. Vénus, qui veille, prend alors soin de l'exciter. La scène se joue comme un nouveau Roman de la Rose R $^{33}$ Amours, Désir, Raison, Loyauté, tour à tour, poussent ou retiennent le héros ; quand Vénus l'a enfin convaincu et qu'il commence à se dévêtir, Loyauté l'arrête. De toutes ses allégories, qui parlent et qui marchent, Vénus est la plus fortement incarnée et elle emporte la cause ${ }^{34}$ : elle esprint son brandon, dont elle embrasa tellement Trö̈lus qu'a pou que la challeur ne le faisoit yssir du sens (p. 90). L'image phallique est sans ambiguïté, elle était annoncée dans le roman par plusieurs descriptions de la fée, embrasée, brandissant son brandon.

L'exemple de Troÿlus est révélateur des difficultés que pose l'image. Face à un sujet inexpérimenté, l'image risque soit de se dissoudre dans la glose, soit fasciner. Dans les deux cas, elle détourne de l'action, de la vie, de la chair, alors que comparé (le corps, la relation sexuelle) devrait

32 Voir la note de G. Roussineau, t. III, 1. III, p. 347.

33 Perceforest doit beaucoup au Roman de la Rose, en particulier pour ce qui est de Nature et de Zéphir, nouveau Genius.

34 Déesse, elle est moins abstraite que Raison et Désir. 
rester prioritaire par rapport au comparant. En ces siècles où la vogue de l'Ovide moralisé est si grande, le propos pourrait surprendre. L'immense plasticité de Perceforest lui permet en fait à la fois de dénoncer les risques de l'image (de l'allégorie entre autres) et d'en montrer la richesse, comme nous le verrons dans la dernière partie. Il faut en effet distinguer deux situations. D'une part, quand l'enjeu est la procréation, nécessaire dans Perceforest qui doit repeupler la Grande-Bretagne et assurer la succession des générations d'Alexandre à Arthur, l'image ne saurait être un euphémisme qui tue le désir, une allégorie dont la glose affadit et diffère l'accomplissement physique: elle doit être clairement érotique, stimulante, purement « utilitaire », Vénus sort son brandon et condamne en quelque sorte l'usage fleur bleue de la rhétorique. Mais lorsque, au-delà des nécessités biologiques, l'Histoire de la Bretagne se construit, non sans jeux spéculaires et analogiques, du paganisme au christianisme, de translatio en translatio, l'image peut retrouver ses lettres de noblesse, comme nous le verrons dans notre dernière partie.

Les dangers d'une polarisation inversée du comparant et du comparé, qui déplacerait le centre de gravité de l'image vers le comparant, se retrouvent, toujours au sujet de l'amour, dans des épisodes de métamorphoses où des images du désir, traditionnelles, sont prises au pied de la lettre. Les transformations d'Estonné en ours, du Tor en taureau à neuf têtes, de Liriopé en levrette, reposent sur des formulations analogiques: le désir du Tor est celui du taureau, la fidélité de Liriopé est celle d'une levrette, Estonné a l'impétuosité d'un ours ${ }^{35}$. La métamorphose prend au pied de la lettre une métaphore implicite $^{36}$. Les personnages sont alors animalisés.

Un épisode du livre V, centré sur Galafur, établit de même un rapport entre l'image, prise au pied de la lettre, et la passion du héros dépossédé de lui-même et «hanté » par la dame. Amoureux, le chevalier monologue: «Dy moy, Amour, est ce par toy que tous vrais amans sont comparez au gentil bracquet qui est de telle nature que quant il est entré en la trace et a sentu l'odeur de la venoison, il

\footnotetext{
${ }^{35}$ Sur ces métamorphoses, voir Fées, bestes et luitons, op. cit., p. 149-ss.

36 Sur les rapports profonds entre métamorphose et métaphore/comparaison, voir Guy Achard-Bayle, Grammaire des métamorphoses. Référence, identité, changement, fiction, Paris, Duculot, 2001, et M. Vuillaume, Grammaire temporelle des récits, Paris, Editions de Minuit, 1990, p. 231, p. 235 et passim. Voir aussi T. Todorov, Introduction à la littérature fantastique, Paris, Seuil, 1970, p. 119.
} 
samble a son maintieng qu'il doive issir du sens? » Dans une longue tirade est alors développé le parallèle entre le brachet et l'amant qui suit sa belle. Plus tard, au moment où Galafur va enfin rejoindre sa dame après l'avoir longuement poursuivie, celle-ci s'enfuit en le comparant à un brachet. Surpris, le chevalier se remet en chemin, glatissant en ses pensees (...) en tant de manieres que se le glatissement eut resonné aussi comme le cuer les ordonnoit affectueusement et en grant ardeur de desir toute la forest en retenty (...). Ainsi chevaucha le bon Gallafur, jusques a soleil esconssant, menant une vie de bracquet gentil, sieuvant la trace en ardant desirier de trouver ce dont il sentoit l'oudeur. A un adversaire qui l'a abattu, il explique: «Je suis homme naturel (...), mais je suis tellement transmué par force d'amour que qui me partiroit mon cuer par le milieu l'en y trouveroit la figure de la plus belle pucelle ». Hanté par la rhétorique amoureuse (à la place du cœur il a la figure de la plus belle pucelle), le héros est quasiment transformé en brachet, alors qu'il ne pense être que comme un brachet.

Dans le même livre V (f. 70v), Passelion croit entendre appeler au secours une cerve poursuivie par quatre chevaliers. Il s'agit de Marmona, revêtue d'une delie vesture couverte de sang. Sur cette pseudo-métamorphose (rationalisée par le vêtement) se greffe une version du Cœur Mangé. L’amour a transformé Marmona en cerve, le prix de sa passion est son cœur. A la fin du livre, cette image de la biche amoureuse a un pendant digne d'un fabliau (f. $289 \mathrm{v}$ ): Dorine, la malmariée, raconte, pour se libérer afin de rencontrer Passelion son amant, qu'elle doit soigner un cerf apprivoisé, qui n'est autre que le chevalier, vêtu d'une peau de bête, ce dont finalement la vieille qui assure la garde de la demoiselle, n'est pas dupe ${ }^{37}$. Dans cet épisode comme dans les aventures de Galafur, le désir transforme le héros en animal, tragique ou comique. L'aveuglement du désir et de la passion conduit à la survalorisation fantasmé du comparant, mortifère: les chevaliers deviennent des animaux, au lieu de se conduire, au pire, comme des bêtes.

Le «Bestiaire d'Amour » que met en place Perceforest est original non dans sa thématique (la « cervitude » d'amour, le brachet, la levrette fidèle), mais dans son fonctionnement. Il prend en effet l'image au pied de la lettre pour la narrativiser grâce à la métamorphose et à ses substituts. Le comparant prend le pas sur le comparé, l'autre (la Dame) devient le soi: le désir provoque des inversions, il est subversif. Prendre ses désirs pour des réalités, et le comparant pour le comparé, est dangereux. Pourtant les métamorphoses du Tor et de Liriopé ont aussi une valeur cathartique, expiatoire,

37 Voir C. Ferlampin-Acher, Fées, bestes et luitons, op. cit., p. 269-270. 
libératrice in fine (la scène se joue à la Maison de Peneance; le vêtement qui permet la métamorphose est une sorte de haire) : la brutalité de l'image, comme dans le cas du brandon de Vénus, permet d'accomplir un désir qui ne se perd peut-être plus dans les mots. L'auteur de Perceforest retiendrait alors de l'image son efficacité, et repousserait la simple contemplation esthétique.

On perçoit alors pourquoi la Beste est évoquée comme un buisson ardent: il sambloit de ceste beste ung buisson par leur couleurs qui entour elle s'entrelachoient (III, II, p. 217) ; ce sembloit a veoir aussi que ung buisson espris de toutes couleurs (1. VI, f. 363). La reprise de cette image, dans deux occurrences aussi éloignées l'une de l'autre, n'est ni accidentelle ni anecdotique. L'article indéfini rend certes incertaine la référence au Buisson Ardent de la Bible (Exode, 3 1-6), mais l'analogie fonctionne bien sur plusieurs points: dans les deux cas il y a une apparition surnaturelle et lumineuse, une voix (celle de Dieu ou, moins mélodieuse, celle de la Beste) se fait entendre, la vision est captivante, Moïse se voile la face car il craint de regarder Dieu, tout comme les admirateurs de la Beste devraient détourner le regard de ce monstre dangereux. Pourtant l'analogie est incomplète et masque l'essentiel : la Beste est mortifère et diabolique. Tout comme l'image qui se forme sur le Buisson Ardent, qui peut révéler Dieu ou la Beste, la rhétorique est ambivalente, celestiel ou terrienne. Cette dualité recoupe partiellement l'opposition entre l'individu, le désir et ses fantasmes qui figent le héros, le piègent, le fascinent, d'une part, et d'autre part, l'homme impliqué dans l'Histoire, la descendance, la généalogie et son dynamisme historique, voire providentiel. Quand on passe d'un système de référence à l'autre, l'image peut être convertie, de la Beste à Moïse. Au début du livre IV, une scène de rêverie, renouvelant la reverdie traditionnelle, associe le plaisir innocent, l'image visuelle et rhétorique (les nuages comparés aux moutons) et un ordre planétaire analogique du monde féodal: l'aer d'entre le ciel et la terre estoit tant meurement entremeslé de vapeurs qu'il sambloit aux regardans que des petites nuees ce fussent blancs moutons pasturans les rais du soleil tres delicieusement et joyeusement; non pas sans plus moutons, car qui prenoit en ce regard plaisir, il y veoit toutes manieres d'hommes et de femmes, de bestes, d'oyseaulx, de valees, de montaignes, de bois, de forests et de signes mervilleux et de horrible façon, les aucuns ayans testes devant et derriere, rechignans les ungs a l'encontre des autres. Tout ce pouoit estre veu en l'aer qui estoit tant mervilleusement paincturé que tous ceulx qui le regardeoient prenoient grant plaisir a le veoir et avoient tresgrant ensonne d'experimenter les merveilles qui pouoient estre veues et figurees (IV, p. 2-3). Dans cette présentation, le style est soutenu, le soleil appelé 
roy des planettes, les personnifications sont nombreuses (le soleil est obedient aux nuées). Si l'image renvoie au corps comme objet de désirs et de fantasmes, elle tue ; si elle renvoie au corps associé à la généalogie et à la procréation, si elle stimule et pousse à agir, à experimenter, elle vivifie: on passe alors de ce Buisson Ardent pervers qu'est la Beste à l'arbre de Jessé.

\section{De la translatio historique à la parabole: Daphné et l'arbre de Jessé}

Perceforest est construit sur un jeu de réflexions spéculaires, qui fait que le principe analogique est omniprésent au niveau de l'inventio comme au niveau de la dispositio. Fondée sur le passage du monde d'Alexandre au monde d'Arthur, du paganisme au christianisme, sa conjointure multiplie les échos entre les matières alexandrine et arthurienne. La translatio (sur le plan idéologique) et la réécriture (sur le plan poétique) ouvrent pour le lecteur des perspectives analogiques d'une richesse étonnante. Par ailleurs, Perceforest raconte l'avènement du christianisme et indirectement d'Arthur.

Parallèlement à ces progrès qui orientent Perceforest, apparaît un autre usage des images. Au lieu de ramener l'objet à la matérialité physique, l'homme à la bête, au lieu de parler aux sens, elles tirent vers l'abstrait, l'immatériel, voire le spirituel. Maronex, dans le livre VI, aimera une demoiselle qu'il n'a jamais vue: "Merveilles est de moy qui aime beauté sans figure ». Dans le même livre, Galafur, à qui revient l'honneur de mettre fin aux enchantements de Bretagne, est à la fois un briseur de statues et le promoteur d'images énigmatiques qui ne peuvent pas fonctionner sur le plan terrien. Iconoclaste, sa mission est d'abattre les œuvres diaboliques au cri de "Mauditz enchantemens, laissez voz deceptions par la vertu du filz de la Vierge”. Le christianisme fera alors advenir des images, des périphrases, des impossibilia, comme le filz de la Vierge, le fils sans pere, le vray crucefié. L'image se libère du corps, aussi bien au niveau de sa thématique (elle ne dit plus le désir et sa chaleur animale, les mères sont vierges et les pères absents), qu'au niveau de son effet (elle ne séduit pas les sens, mais l'esprit). Le fantasme laisse la place à la vision, souvent onirique et prémonitoire. L'image alors s'accompagne d'une

glose: Vénus refusait d'élucider la comparaison avec la raiere, la Reine Fée ou Dardanon ne se soustrairont pas à ce devoir, annonçant en cela les preudommes de la Queste del Saint Graal. Souvent le commentaire est explicite. Dardanon explique à Perceforest une de ses visions et développe la senefiance du sceptre qui doit signifier la verge de justice: (...) le roy la doit tenir droicte en sa main 
pour luy monstrer estre appareillié en tout temps de corrigier et faire justice des mauvais, ainsi que le maistre en l'escole doit tousjours tenir sa verge droicte pour plus estre appareillé de corriger ses escoliers (II, I, p. 236-ss). Quand la glose ne paraphrase pas, l'avenir éclaircit tout, comme l'explique à Passelion la Déesse des Songes: "Votre songe a grant seigniffiance, car advenir convient ce qui est ordonné par les menistres du dieu de nature sur chascune personne (...). Les journees d'ores en avant sont toutes taillies qui te exposeront ton songe de fait mieulx que se je te disoie par parolles ». Fondé sur un principe d'analogies, le songe se présente alors comme une métaphore in absentia appelée à devenir dans l'avenir (de Perceforest ou, plus largement, dans l'avenir arthurien) une comparaison.

A titre d'illustration, trois songes me retiendront plus particulièrement: dans le livre II, ceux de Gadifer (II, I, p. 85-86) et de Perceforest (II, I, p. 232-ss) et dans le livre V celui de Galafur (f. 272-ss).

Dans le premier songe, Gadifer, endormi dans son lit, se voit nu, dans un beau verger ; il éprouve une agréable impression de chaleur, de confort, de sécurité, s'étend voluptueusement sur sa couche, et se met à chanter: adont print a regarder par dessus luy et voit tout en chantant ung lorier qui le couvroit de ses branches (II, I, p. 85). Un preudomme, dans l'arbre, tient un rolle de parchemin qui lui conseille de réfréner son chant, car la joie dure peu. Le preudomme disparaît, et un lion, un griffon et un léopard mettent en pièce le corps du roi. La reine, à son réveil, lui suggère de s'en remettre à Dieu et ajoute: «C'est exemple a vous chastoier que ne montez en trop grant orgueil » (II, I, p. 86) .

Plus loin, c'est au tour de l'autre grande figure royale du roman d'avoir un songe prémonitoire (II, I, p. 233). Perceforest, dans une forêt, se voit comme un roi dénué de toute autorité: "Aprés ce regarday par la forest et vey qu'il y avoit plenté d'estocq ${ }^{38}$ dont les arbres avoient esté coppez pour ce qu'ilz estoient brehaignes et de nulle valeur et qu'ilz empeschoient aux bons. Mais quant j'euz bien considéré les estocz, je vey apertement que puis ung pou de temps et par deffaulte de forestier les estocz avoient jecté grans jectons qui commençoient a fourmener les bons arbres, sy que les branches en commençoient a sechier par l'empeschement des mauvais jectons. Et encores croissoit entour les bons arbres ung mauvais arbre qui estoit appellé yerre, qui s'envelopoit entour les bonnes branches qui estoient demourees et leur tolloit le soleil, sy ne pouoient fructifier $»$. Des oiseaux gentilz veulent se

38 Souche, racine, bûche (on le trouve dans le Joli Buisson de Jonece de Froissart, éd. A. Fourrier, Genève, Droz, 1975, v . 4909). 
poser près du roi, mais des chats-huants et des corbeaux les en empêchent. "Quant j'euz ce regardé, je me pensay que en ce fait avoit grant signifiance » (II, I, p. 235). Le roi voit alors un vieil homme qui ressemble à Dardanon et qui lui fait signe de venir lui parler. Dardanon glosera en effet plus tard ce rêve envoyé par Dieu et expliquant, selon lui, les devoirs royaux (p. 236). Dans cette représentation du roi forestier, le lierre et les oiseaux symbolisent les menaces qui pèsent sur le royaume: les chashuans c'est a dire les losengeurs et les traytres. Cette glose est nourrie d'images diverses, qui relancent le processus métaphorique, assimilant, ce qui n'était pas le cas dans le songe, le roi à un maître d'école, et introduisant la référence biblique «Vé de la terre dont le prince est enfant » (II, I, p. 243).

Dans le livre V (f. 272-ss), Galafur, endormi, se sent emporté auprès de la Reine Fée, à qui il raconte le rêve qu'il a eu précédemment dans le temple des Songes. La double distanciation onirique s'explique par la gravité et la complexité de cette vision construite sur un triptyque au terme duquel Galafur reçoit de la Reine Fée l'écu marqué de la Croix du Christ et apprend l'emplacement des chroniques qui serviront de base au roman. Dans un premier temps, la croissance d'une forêt illustre la restauration de la Bretagne, puis le roi voit l'intérieur de son corps, et en particulier ses veines, en transparence et les trois gouttes de sang qu'il donne à la Reine deviennent trois enfants, et enfin, à peine voilée, après la reconstruction de la Bretagne, sont évoqués Zéphir, une nouvelle décadence et une renaissance, que suit l'évolution de la taille du roi, qui diminue et grandit à nouveau, pour la plus grande joie du rêveur qui se réveille. La liaison entre les trois parties est assurée par la conscience du rêveur et par la continuité de la thématique de l'arbre, du laurier, à la ramification des veines, puis à la forêt ${ }^{39}$, qui représente le roi, confondu avec son royaume et sa descendance ${ }^{40}$. Plus qu'une métaphore ou une comparaison, nous avons ici une analogie filée et glosée, un développement de type allégorique, que nous appellerons parabole ${ }^{41}$.

\footnotetext{
39 Toutes les vaines qui par mon corps s'espardoient avoient commencement a lui et prendoient sang et doulceur pour tout le corps arouser.

${ }^{40}$ La similitude entre le végétal et le corps n'est pas surprenante: nous avons tous un tronc.

${ }^{41}$ Pour une définition de l'allégorie comme métaphore filée et glosée, voir A. Strubel, "Grant senefiance a". Allégorie et littérature au Moyen Age, Paris, Champion, p. 19-ss. Notons que la personnification qui caractérise de nombreuses allégories (comme dans Le Roman de la Rose) est ici inversée, puisque le roi sert de comparé et non de comparant. Nous sommes plus du côté de La Queste del Saint Graal que du Roman de la Rose, d'où notre emploi du terme "parabole" (sur La Queste del Saint
} 
Dans le premier rêve, le laurier peut sembler purement décoratif; il peut aussi bien être un emblème, transparent, de la royauté en paix et victorieuse. L'essentiel du songe paraît ailleurs, dans le démembrement du corps royal par des animaux. Ce symbolisme animal est banal ${ }^{42}$, tout comme l'identification du royaume au corps du roi. Par ailleurs le lecteur arthurien n'est pas étonné par le vieillard dans l'arbre ${ }^{43}$. Le rolle n'est guère plus surprenant: quelques chapitres plus tôt, Lyonnel a trouvé dans un chêne un rolle qui l'encourage après ses déceptions lors du bain des fées (II, I, p. 194). L'impression de familiarité peut venir aussi du cadre onirique, traditionnel, et surtout peut-être du fait que cette scène combine deux motifs iconographiques fréquents, tous deux liés à un arbre dynastique: d'une part l'arbre lié au rêve, comme dans le second songe de Nabuchodonosor (Daniel, 4) ${ }^{44}$, dans l'épisode de l'arbre de Jessé (Isaïe, XI, 1-3) ${ }^{45}$ ou bien encore dans le songe d'Astyage ${ }^{46}$, et d'autre part le maître de l'arbre, le «dominus arboris » ${ }^{47}$ qui présente et soutient l'arbre généalogique ou l'arbre de

Graal comme parabole, voir A. Strubel, La rose, Renart et le Graal. La littérature allégorique en France au XIIIe siècle, Paris, Champion, 1989, p. 269-ss).

$42 \mathrm{Il}$ remonte à Geoffroy de Monmouth: voir C. Daniel, Arthurianisme et littérature politique, thèse soutenue sous la direction de J. Cl. Thiolier, Paris, XII, 2002, t. I, p. 321-ss.

${ }^{43}$ On voit bien un enfant dans un arbre dans la deuxième Continuation de Perceval et dans Durmart, et une demoiselle dans Cristal et Clarie.

44 Daniel, 4 1-33. On en trouvera une représentation tirée du Miroir de Salut Humain du manuscrit de Marseille BM ms 89 f. 24v dans Le Moyen Age en lumière, sous la direction de J. Dalarun, Paris, Fayard, 2002, p. 99.

45 Voir A. Strubel, "Allegoria in factis et allegoria in verbis », dans Poétique, 1975, n²3, p. 342-357 où l'on trouvera des commentaires concernant l'arbre de Jessé. G. Duby consacre une page et une illustration à l'arbre de Jessé, Le Moyen Age, 1140-1280, Skira, Genève, 1984, p. 30-34.

46 Selon Hérodote, Astyage rêva que du ventre de sa fille Mandane sortait un torrent qui couvrait l'Asie entière: pour ses mages, ce rêve annonce la future domination des Mèdes sur l'Asie entière. Inquiet pour son trône, Astyage choisit pour époux à sa fille un Perse de condition inférieure. Un second songe lui fait voir une vigne sortant du ventre sa fille et couvrant l'Asie, ce qui, pour les mages, confirmerait l'hégémonie mède. Quand l'enfant, Cyrus, naît, Astyage le remet à Harpage pour qu'il le tue. Comme dans le songe de Perceforest l'enjeu est le pouvoir. Ce récit est très connu au Moyen Age. On le trouve par exemple dans le Speculum Historiale de Vincent de Beauvais, avec un renvoi à Eusèbe. Voltaire, dans son Dictionnaire Philosophique, n'a pas manqué de s'en moquer. Merci à Denis Hüe de m'avoir signalé cet épisode. Nous verrons plus loin que même si le songe d'Astyage ne met pas en scène un arbre mais une vigne, il a souvent été associé à Jessé et Nabuchodonosor (voir la note 47).

47 Voir Le Moyen Age en lumière, op. cit., p. 107-ss "la parenté en arbre". 
consanguinité, et en cautionne la vérité par son autorité ${ }^{48}$. Dans ce premier rêve se retrouveraient donc un symbolisme animal à la Geoffroy de Monmouth et un arbre, évoquant des représentations généalogiques. La nature de cet arbre, un laurier, s'expliquera peut-être plus loin.

Dans le deuxième songe, on passe de l'arbre à la forêt. Ce rêve sert de pendant au premier, dont il n'est pas éloigné dans le roman: les deux rois Perceforest et Gadifer, dans le livre II, évoluent parallèlement. La restauration du royaume, représentée par la forêt et la croissance des arbres, clarifie le rapport analogique entre la royauté et les arbres, rapport qui, dans le premier rêve restait implicite.

Le troisième rêve, beaucoup plus complexe, reprend la conjonction du songe, du roi, des estocs dont la pousse est difficile, des cycles de croissance et de décadence. Il introduit un élément nouveau, qui va peut-être nous permettre de comprendre la mention du laurier dans le premier rêve. Dans le songe de Galafur est en effet décrite la métamorphose d'arbres en humains :

« Les forestz estoient petites et basses car les plus granz estocz n'avoient point deux piez de hault, toutesfois estoient ilz vers et desirans de venir. Moult m'esmerveillai de ceste chose et prins fort a regarder, et tant qu'il me fu advis que je les veisse croistre en haulteur et leurs rains estendre a l'environ (...). Un pou aprez fu la chose plus merveilleuse car la ou je regardoie aucuns arbres croistre plus hault que celz d'entour, il me fu advis que je veisse les couppiers muer en chief d'hommes petit a petit et en après ses brances en bras et en jambes. Sous la conduite du plus grand arbre, ils construisent des villes sicque en pou d'heure je vey toute la Grant Bretaigne redifiee et si vis plenté de chevalerie chevaulchier par le païs dont je ne gardai l'heure que tous s'en vindrent a l'entour moi. Si me firent roi et seigneur dessus eulz ».

Dans un premier temps, cette forêt qui se met en marche nous rappelle la grande forêt de Birnam de Macbeth $^{49}$, que Shakespeare a emprunté aux chroniques d'Hector Boyce (1527), les Scotorum Historiae. Perceforest aurait pu puiser aux mêmes sources latines que Boyce. Il me semble néanmoins

48 Le songe d'Astyage a été mis au Moyen Age sur le même plan que ceux de Jessé ou de Nabuchodonosor. Dans le Speculum Humanae Salvationis (édité en 1470 en Hollande) chaque bois gravé présente deux compartiments: 1'un juxtapose l'annonce de la naissance de Marie et le songe d'Astyage ; immédiatement après, un autre regroupe la naissance de Marie et l'arbre de Jessé ; plus loin, le rêve de Nabuchodonosor est associé à la Crucifixion. Jessé et Astyage entrent en résonance ; les songes de l'arbre renvoient, comme dans le commentaire de la Reine Fée, au Fils de la Vierge, au Vray Crucifié. Voir la reproduction des bois gravés et du texte dans The Mirror of Salvation, Duquesne University Press, 2002.

49 Great Birnam wood to high Dunsinane hill / Shall come against him (I, 4). 
que c'est ailleurs qu'il faut chercher. Cette métamorphose prend au pied de la lettre l'analogie, banale, de l'arbre et de l'homme, étayée par les vocabulaires moderne («rejetons ») et médiéval (stemmata, stirps, fruit $\left.^{50}\right)$. Cette analogie est double : anatomique, elle marque la similitude morphologique entre l'homme et l'arbre (qui ont tous deux des troncs) et d'autre part, elle est généalogique, et représente l'individu et sa descendance par le tronc et les branches. Dans ce rêve, les deux coexistent, puisque l'arbre se transforme en humain d'une part et d'autre part le songe s'achève sur un aperçu de la lignée du roi. Or pour la transformation d'arbre en homme, les Métamorphoses d'Ovide me paraissent offrir références intéressantes, permettant qu'une unité signifiante soit constituée par le laurier du premier rêve, les arbres qui se mettent en marche et constituent une armée conquérante, la généalogie de Galafur, et l'ouverture du discours de la Reine Fée sur le Christ et la Vierge. L'auteur connaissait certainement la fable de Daphné, diffusée largement par les Ovide moralisé, tant en vers qu'en prose ${ }^{51}$. Le songe modifie certes le processus métamorphique ovidien : au lieu que ce soit l'humain qui devienne végétal, c'est l'inverse ; par ailleurs dans le songe de Galafur ce sont des hommes qui se transforment, et non une figure féminine, comme la nymphe, mais le terme hommes peut être pris au sens générique, et non sexuel. Malgré ces différences, il n'est pas impossible que le modèle de ces arbres qui deviennent humains soit Daphné :

- la description que le roman médiéval donne de la transformation correspond à ce que l'on peut lire chez Ovide et dans l'Ovide Moralisé (en particulier pour ce qui est des bras et des branches);

- l'inversion du sens de la métamorphose n'est pas surprenant : la «mutation », qui doit respecter l'œuvre de Dieu, est en général pensée comme provisoire et réversible au Moyen Age ;

- les métamorphoses en arbre sont rares dans la littérature médiévale, et la renommée de Daphné suggère qu'il n'était concevable qu'un lettré comme l'auteur de Perceforest puisse évoquer une telle mutation sans penser à elle ;

- le laurier du premier rêve ${ }^{52}$ se comprend mieux ;

\footnotetext{
${ }^{50}$ Dans Perceforest, c'est en général en terme d'arbres que se rêve la généalogie: les enfants sont les fruits, on parle de la rachine de Darnant pour désigner son lignage (1. VI, f. 329v).

51 Sur Daphné, voir Y. Giraud, La fable de Daphné, Genève, Droz, 1969. Pour le Moyen Age voir p. 83-154.

52 Même si l'Ovide moralisé en vers n'est en général pas intéressé par la dimension étiologique des métamorphoses ovidiennes (cf. le mûrier de Pirame et Tisbé par exemple, voir C. Ferlampin-Acher, "Pyrame et Tisbé: le vert paradis des amours enfantines et la mort des amants", Lectures d'Ovide, publiées à la mémoire de Jean-Pierre Néraudau, textes réunis par
} 
- Daphné, figure de la chasteté (les moralisations ovidiennes insistent sur ce point ${ }^{53}$ ), permet de saisir un lien entre les trois temps du rêve de Galafur : la seconde partie évoque, de façon imagée, sans qu'il soit question de sexe, la conception de trois enfants ; la dernière, généalogique, conduit au Chevalier Vierge, (prémonition de Galaad), qui, à la fin de l'épisode, recevra l'écu marqué d'une croix christique et apprendra à combattre au nom du Fils de la Vierge, le Christ ;

- le songe de Jessé qui sert de modèle iconique au premier rêve se trouve remotivé, puisqu'il annonce la généalogie de Jésus et Marie.

Ces trois rêves qui développent sur le mode allégorique de la parabole l'histoire d'une dynastie et l'avènement providentiel du christianisme, reposeraient sur un travail autour de l'arbre. En plaçant un laurier au seuil du premier rêve, l'auteur annonçait à la fois la royauté arthurienne triomphante, et l'avènement de la Vierge et de son Fils, il combinait à la fois le celestiel et le terrien. L'arbre permettait ainsi de « conjoindre » paradoxalement et idéalement la lignée (nécessaire à la translation imperii) et la chasteté (célébrée dans une perspective providentielle) . A la place d'une image qui s'impose et séduit d'emblée; le rêve allégorique propose une constellation d'analogies qui se construisent petit à petit (laborieusement ?) et stimulent la réflexion, tandis que sur le plan thématique, on est passe du désir à une conception de l'amour qui combine idéalement le lignage et la chasteté. Dans ce système analogique, la métamorphose des arbres en hommes constitue une clef essentielle.

Cette importance de la "mutation » végétale est confirmée par l'attitude de la Reine Fée qui, commentant l'ensemble du rêve, omet de parler de la transformation des arbres. Galafur s'en étonne et insiste avec curiosité: "Dites moi pourquoi il me fut advis que les arbres des forestz se muoient en hommes. -Par ma foi, chier niepz, dist la dame, cela sert pou au fait fors a la fantasie de la regardeure, et si devez sçavoir car qui pourroit veoir le grant desirier que la terre et le païs de soi (soi) repeupler, il

E. Bury, Paris, Les Belles Lettres, 2003, p. 115-148), dans le cas de Daphné, c'est bien sur les origines du laurier que s'ouvre le récit médiéval (éd. C. de Boer, Johannes Müller, Amsterdam, 4 tomes, 1915-1936, 1. I, v. 2737-2738). Par ailleurs la chaleur qui est mentionnée au début du rêve de Gadifer, en même temps que le laurier (II, I, p. 85-86), permet certes de faire contraste entre le plaisir éprouvé par le dormeur au début du songe et la fin du rêve, pénible, mais renvoie peut-être aussi au laurier qui aime la chaleur du soleil, tout comme Phébus aime Daphné (conformément à la glose naturelle donnée par l'Ovide moralisé 1 . I, v. 3068-ss).

${ }^{53}$ Voir P. Y. Giraud, op. cit., p. 94-ss. La base de cette lecture de la métamorphose de Daphné est l'allégorisation d'Arnoul d'Orléans. 
me fust advis qu'il n'eust sur terre arbres ne pierre qui ne se muast en figure d'homme ou de femme et pour ce vous sambloit ilz que les arbres devenissent hommes ». Cette réticence contraste avec l'ampleur de la glose proposée par une reine qui ne ménage pas ses efforts. Selon elle cette métamorphose ne serait qu'une fantasie, un des ces ornements dont nous avons vu justement que Perceforest ne les prise guère. Le lecteur peut en douter.

En fait, le silence de la Reine est significatif, non du caractère anecdotique et décoratif de la métamorphose et de l'image qui la sous-tend (l'homme est comme un arbre), mais au contraire de son caractère «crucial ». La Reine refuse de commenter la métamorphose d'une part parce que cet élément est celui, des trois rêves, qui ressortit le plus clairement à la mythologie païenne, ce qui pourrait paraître peu conciliable avec son projet d'annonce providentielle ${ }^{54}$, et d'autre part et surtout, parce que cette métamorphose concerne finalement les mystères ultimes du christianisme en introduisant, par le biais de Daphné, la figure de la Vierge ${ }^{55}$.

Le seul argument que la Reine finit par présenter pour justifier rapidement la métamorphose confirme d'ailleurs l'hypothèse de l'inspiration ovidienne. En effet elle affirme que la volonté de repeupler le pays pourrait suffire à expliquer qu'il n'eust sur terre arbres ne pierre qui ne se muast en figure d'homme ou de femme: la métamorphose des pierres, qu'elle associe à la métamorphose des arbres, peut renvoyer à l'histoire de Deucalion, épargné par Jupiter lors du Déluge, qui crée un nouveau genre humain en jetant des pierres derrière son dos. Le rapprochement est possible: deux légendes ovidiennes (Daphné et Deucalion), voisines dans ce vaste ensemble que sont les Métamorphoses, seraient mises en relation ${ }^{56}$. L'histoire de Deucalion, associée au Déluge et au restorement /D'umain

\footnotetext{
${ }^{54}$ La translatio studii relativise cependant fortement cet argument.

${ }^{55}$ Dans l'Ovide moralisé en vers, Daphné est explicitement associée à la virginité (1. I, v. 3075-ss) et à la Vierge Marie (v. 3215-ss).
}

56 Dans l'Ovide moralisé en vers on trouve dans le livre I à la fois Deucalion (v. 1945-ss) et Daphné (v. 2737-ss). L'histoire de Cadmus, très connue au Moyen Age (Le Roman de Thèbes, aux origines du genre romanesque médiéval, y fait référence), est voisine de celle de Deucalion et peut avoir aussi suggéré l'idée de la métamorphose de pierres en hommes: Cadmus sème des dents de dragon qui se transforment en soldats ; ainsi aurait été fondée Thèbes. Des pierres aux dents, le passage se fait sans problème, puisque dans l'histoire de Deucalion, l'ordre divin était d'abord de semer les os de la grande mère, ce qui s'avère être en fait une image pour désigner les pierres de la terre. Si les os peuvent permuter avec des pierres, les dents le peuvent aussi. 
lignage dans la glose moralisée en vers (v. 2019-2020) n'est de plus pas étrangère à la vision que le rêve de Galafur et plus largement l'ensemble de Perceforest donnent de l'histoire et de la préhistoire arthuriennes. Dès lors, les arbres qui deviennent humains, tous comme les pierres, peuvent être d'inspiration ovidienne, et rappeler Daphné, héroïne de la métamorphose végétale la plus connue au Moyen Age.

Ces arbres peuvent néanmoins aussi évoquer Orphée (Métamorphoses, XI, v. 1 et X, 3) s $^{57}$ à la fois parce que son chant fait marcher les arbres et parce qu'à sa mort, Bacchus, pour punir les femmes thraces de l'avoir tué, les transforme en arbres. Si les arbres renvoient à Orphée, les pierres peuvent alors évoquer la transformation en rochers des Propetides (Ovide, Métamorphoses, X, v. 238-ss, Ovide Moralisé en vers, 1. X , v. 912-928), chantées par Orphée. Si l'histoire de Daphné est conjointe à celle de Deucalion, celle d'Orphée l'est à celle des Propetides, et il est difficile de privilégier une piste à l'autre : dans les deux cas, la métamorphose en pierre à lieu dans le même sens que dans le discours de la Reine Fée, alors que la mutation végétale est inversée (de l'humain vers l'arbre). On notera d'ailleurs que dans la version médiévale en vers Vénus métamorphose les Propetides en ymage de pierre ou de fust (1. X, v. 927), alors que chez Ovide elles ne sont changées qu'en pierres (1. X, v. 243) : comme dans le discours de la Reine Fée, les métamorphoses en arbres et en pierres/ d'arbres et de pierres sont rapprochées.

La polysémie des gloses ovidiennes rend vraisemblable le renvoi à Orphée et Daphné (et, associés, à Deucalion et aux Propetides): ces figures posent en effet toutes pour le glossateur médiéval le problème de la virginité et de la generation (au sujet des cordes de la lyre orphique 1. X, v. 2600-ss, des arbres 1. X, v. $3052^{58}$, de Deucalion 1. I, v. 2159-ss). Le récit de la mort d'Orphée rapproche, comme l'histoire de Daphné, la métamorphose en arbre et la chasteté : les ménades, qui, furieuses, enflammées de désir pour Orphée, le tuent, sont punies par Phébus qui les métamorphose en arbres; elles sont

\footnotetext{
${ }^{57}$ Le talent d'Orphée «mobilise » arbres et animaux. Parmi les arbres émus, figure le laurier virginal, associé à Daphné (Métamorphoses, éd. et trad. G. Lafaye, Paris, Les Belles Lettres, 1928, rééd. 2002, 1. I, v. 92, Ovide moralité en vers, 1. X, v. 3053).

${ }^{58} \mathrm{Au}$ sujet des arbres, Ceulz qui miex font a desclairier / Li pins et l'ierre et le lorier, / Qui ont pardurable verdure, / Notent ceulz qui metent lor cure / En garder pardurablement / Lor virginité netement (1. X, v. 3052-ss). La polysémie du symbole médiéval est nette: le lierre parasite qui empêche la croissance dans le rêve de Perceforest représente aussi la vertueuse virginité.
} 
l'exacte antithèse de Daphné ; dans les deux cas la métamorphose en arbre contraint à la chasteté, Daphné en s'en félicite, les Ménades moins.

Il est certes difficile de mesurer l'impact des références que j'évoque. Nous ne sommes pas dans l'Ovide Moralisé et la Reine refuse d'en dire plus. Par ailleurs, s'il est certain que la virginité constitue l'un des vecteurs de cohésion de ce rêve qui aboutit à la Vierge, et prend la suite d'un arbre qui ressemble, dans le premier rêve, à un arbre de Jessé où se lit la généalogie de Marie, le thème est trop présent dans 1'Ovide moralité pour permettre d'identifier définitivement telle ou telle métamorphose (Daphné est loin d'être le seul symbole de la chasteté dans ce texte obsédé par les images mariales). Ces réserves émises, il me semble néanmoins que ces trois songes, loin d'être disjoints, forment un système cohérent, autour du rêve de l'arbre, qui est à la fois arbre généalogique (ces songes témoignent d'un puissant souci dynastique) et arbre de la virginité (Daphné, la Vierge, Jessé), et qui annonce peut-être aussi l'Arbre de la Croix ${ }^{59}$ puisque la glose de la Reine nous conduit au « Vrai Crucifié ». Le devenir du royaume est pensé en termes féodaux et chrétiens et la sexualité est gommée par les références à l'arbre, à la métamorphose, et par le don imagée des gouttes de sang, plus christique qu'érotique. L'image tend vers la parabole, elle renvoie aux mystères divins, elle ne parle pas à la sensualité mais à l'intelligence (les difficultés que nous avons à lui construire un sens prouvent bien que, contrairement à l'image de la raiere et du fruit que nous avons rencontrée plus haut, elle ne vise pas l'immédiateté). L'image vaut parce qu'elle résiste à l'évidence et à sa séduction, elle ne vaut que par le décryptage qu'elle nécessite. On rejoint alors la parabole, qui développe un double sens qui s'élabore peu à peu, difficilement, artificiellement. Ces trois rêves aboutissent en même temps au Fils de la Vierge et à la révélation par la Reine de l'emplacement des chroniques que Galafur devra poursuivre et qui serviront de base au rédacteur de Perceforest. Ce retour sur l'écriture rappelle que le premier rêve doublait l'événement d'un rolle, d'une commentaire. La pratique parabolique suppose finalement une pratique métalinguistique, un retour du texte sur lui-même, un « enroulement » /enrollement du texte sur lui-même (si l'on me permet cette image).

59 Pour une représentation de la Croix associée à un arbre véritable, l'Arbre de Vie, voir Le Jardin de Vertueuse Consolation, Bruges, vers 1474, BnF. fr. 1026, f. 9v, reproduit dans M. Smeyers, L'art de la miniature flamande du VIIIe au XVIe siècle, trad. franç. Tournai, La Renaissance du Livre, 1998, p. 412. Sur l'arbre et la croix, voir R. Bauerreiss, Arbor vitae. Der Lebensbaum und seine Verwendung in Liturgie, Munich, 1938. 
Cette valorisation de l'allégorique ne surprend finalement pas chez un auteur qui connaît et utilise le Roman de la Rose (Zéphir avatar de Genius et la plaintive Nature lui doivent beaucoup). D'autant que le XIVe et le XVe siècle (dates de l'écriture et de la réécriture supposées de ce roman) sont marqués par un renouveau allégorique. Perceforest serait alors à la croisée de l'Ovide Moralisé, du Roman de la Rose, voire de Christine de Pizan ${ }^{60}$. Sa prédilection pour l'allégorisme tardif tout comme son désintérêt pour les comparaisons topiques, les métaphores séduisantes, et le symbolisme animal à la Geoffroy de Monmouth seraient bien de son époque.

\section{Conclusions}

- L'analyse de ces quelques épisodes permet de faire des propositions quant à l'art poétique mis en pratique dans Perceforest. La comparaison topique est discrète: c'est affaire de mode, c'est aussi parce que la rhétorique est condamnée comme deception, illusion. Les dangers de l'image sont associés à la fascination esthétique et à la projection d'un désir autarcique. L'image tue, et non la lettre. Elle brouille les catégories, inverse le dedans et le dehors, le comparant et le comparé. Le risque majeur est de prendre au pied de la lettre ce qui n'est que similitude. La métaphore, qui omet le comparé, est, plus que la comparaison, qui fait figurer à la fois le comparé et le comparant, à la base de cette condamnation. Passe en effet encore de glatir comme un chien quand on est amoureux: il est en revanche plus risqué de devenir un vrai taureau. Perceforest souligne de plus l'inefficacité de l'image, impuissante devant l'indicible de la merveille et il renonce à l'euphémisme amoureux et préfère l'image sensuelle, visuelle, parlante, qui peut être un vigoureux stimulant. Mais c'est surtout comme parabole que, glosée, l'image trouve ses lettres de noblesse : elle porte le sens de l'histoire, stimule le lecteur à jouer, non de ses sens, mais de son intelligence, à décrypter, à translater, comme le montre le songe de l'arbre. Finalement Perceforest nous invite à passer de l'image païenne, adorée et sensuelle, au Verbe chrétien, à l'énigme ineffable du fils de la vierge, qui, loin d'enchaîner l'homme à la terre et aux désirs, loin de le rouler dans

\footnotetext{
${ }^{60}$ Dans son Advision Cristine, Christine de Pizan évoque l'arbre d'or des Troyens, en relation avec la translatio imperii (éd. C. René et L. Dulac, Paris, Champion, p. 7-9): à travers les gitons de l'arbre d'or qui furent plantez en plusieurs par se peut entendre plusieurs des parens du roi Priant de Troie qui se transporterent en divers païs, dont sont venus plusieurs nobles generacions comme les Rommains et autres, et meïsmes en France. Elle évoque aussi une vergete sur cet arbre qui puet signifier le lignaige Nostre Dame (p. 8), ainsi que la menace du lierre. C'est bien le même imaginaire, le même jeu allégorique et le même enjeu idéologique que dans Perceforest.
} 
la boue comme le fait Zéphir, l'élève à la pureté celestiel. Au-delà des dangers de la métaphore, se révèlent donc les richesses de la parabole et des figures allégoriques, qui proposent des gloses, et donc ne confondent pas comparé et comparant. Affaire de mode aussi peut-être que cette poussée allégorique dans Perceforest, mais pas seulement, car elle renvoie plus profondément à la littérature et aux mystères chrétiens les plus profonds. Chronique, Perceforest se méfie donc des images; roman, il en avoue la nécessité ${ }^{61}$; Evangile (sa fin rejoint 1'Evangile de Nicodème), il en expérimente la vertu. A lire Perceforest, on éprouve finalement les joies et les vertiges de l'analogie, dans ce qu'elle a de risqué, de stimulant, de dépaysant et de fécond ${ }^{62}$.

- Parallèlement à ces valorisations diverses de la comparaison, de la métaphore et de la parabole, on peut noter des préférences sémantiques quant au comparant. La comparaison topique fait appel à des domaines divers, lis, lumière, tonnerre ; éclatée, elle ne fait sens que par rapport à des pratiques héritées et ne forme pas vraiment de système propre à Perceforest. Plus original peut-être, la référence animale me semble dépréciée dans Perceforest: elle renvoie le plus souvent à la bestialité, à la métamorphose et à la métaphore confusionnelles; le «bestiaire divin » me paraît peu présent. En revanche le végétal est valorisé. Cette dichotomie correspondrait à l'idée qu'il faut dépasser le désir physique, les unions charnelles marquées par l'animalité (inévitables, nécessaires, nombreuses et plaisantes pour le lecteur quand le facétieux Zéphir ou Passelion entrent en scène), par une génération imaginée comme asexuée, purifiée, sur le mode de la bouture, de la ramification, du végétal. La célébration de la chasteté, dans l'esprit de la Queste del Saint Graal, se fait alors, comme souvent dans ce roman, par le biais de la parabole, onirique et glosée.

- Finalement, Brunet Latin aurait aimé Perceforest, lui qui conseille: Mais comment que ta parleure soit, ou par rime ou par prose, esgarde que ti dit ne soient maigre ne sech, mais soient replain de jus et de sanc, c'est a dire de sens et de sentence. Garde que ti mot ne soient nices, ains soient grief et de grant pesantour, mais non mie de trop grant, ki les fesist atrebuchier. Garde qu'il n'aportent laidures nules,

\footnotetext{
${ }^{61}$ Le moine de l'abbaye de Saint Landelin à Crespin, qui traduit le manuscrit, l'enjolive et ajoute ce qu'il faloit a l'oeuvre vesture de parolles; les matières sont colorees d'armes et d'amours, dont le conte luy pria moult qu'elle en fust aornee (t. I, p.124).

62 Comme le note M. Stanesco, «Parole autoritaire et accord des semblances » dans La Queste del Saint Graal», dans Mélanges Philippe Ménard, op. cit., p. 1273, "l'analogie est aujourd'hui considérée plutôt comme un moyen honteux de raisonnement", alors qu'au Moyen Age elle est un "moyen d'argumentation" et une "véritable forme de démonstration".
} 
mais la bele coulours soit dedens ou dehors, et la science de rectorique soit en toi peinturiere, ki mete la couleur en risme et en prose. Mais garde toi de trop poindre, car aucunefois est couleur a eschiver couleur (III, 10). 